\title{
A new difference method for the singularly perturbed Volterra-Fredholm integro-differential equations on a Shishkin mesh
}

\author{
Musa Çakır (D), Baransel Güneş*(i) \\ Van Yüzüncü Yll University, Department of Mathematics
}

\begin{abstract}
In this research, the finite difference method is used to solve the initial value problem of linear first order Volterra-Fredholm integro-differential equations with singularity. By using implicit difference rules and composite numerical quadrature rules, the difference scheme is established on a Shishkin mesh. The stability and convergence of the proposed scheme are analyzed and two examples are solved to display the advantages of the presented technique.
\end{abstract}

Mathematics Subject Classification (2020). 45J05, 65L11, 65L12, 65L20, 65R20

Keywords. difference scheme, error estimate, Fredholm integro-differential equation, singular perturbation, Shishkin mesh, Volterra integro-differential equation

\section{Introduction}

Volterra-Fredholm integro-differential equations (VFIDEs) have arisen in different areas of science and engineering. Their implementations can be found in electrostatics [14], biological models [37], atomic physics [20], astronomy [16], potential theory [17], fluid dynamics [2] and electromagnetic theory [32] (see, also references therein).

There are many researchs about VFIDEs in the literature. The existence, uniqueness and stabiliy of them were debated in [6,25,27,28]. Furthermore, different numerical and semi-analytical methods have been proposed by many scholars. Adomian decomposition method and its modified versions were used in [12,17, 18, 24, 29]. Variational iteration method was applied in [41]. Bernstein polynomials method was suggested for neutral type VFIDEs in [20]. Legendre collocation matrix method was developed for high-order VFIDEs in [39]. Homotopy perturbation method was used in [15,34]. By using trapezoidal quadrature rules, the finite difference method was considered in [34]. Orthonormal Bernstein and Block Pulse functions method were presented in [35]. Shifted Jacobi spectral collocation method was performed to multi-dimensional VFIDEs in [10]. Nyström discretization approach was introduced in [13]. Galerkin method was carried out by using Legendre basis functions in [14,23]. Nonlinear type VFIDEs were examined with the help of Chebyshev cardinal functions in [21]. Tau method was given for one and twodimensional VFIDEs in $[36,37]$. Collocation method with Boubaker wavelet functions was

\footnotetext{
*Corresponding Author.

Email addresses: cakirmusa@hotmail.com (M. Çakır), baranselgunes23@gmail.com (B. Güneş)

Received: 28.06.2021; Accepted: 22.11.2021
} 
proposed for high-order VFIDEs in [32]. Integral collocation approximation was employed for high-order VFIDEs in [1]. The other techniques have been described in a series of papers $[2,7,8,16,19,31,38]$. The above-mentioned studies were only concerned with the regular cases (i.e. as the lacking of the singularity).

In this paper, we consider the following initial value problem of singularly perturbed Volterra-Fredholm integro-differential equation (SPVFIDE):

$$
\begin{gathered}
L u:=L_{1} u+\int_{0}^{x} K_{1}(x, t) u(t) d t+\lambda \int_{0}^{l} K_{2}(x, t) u(t) d t=f(x), x \in I=[0, l], \\
u(0)=A
\end{gathered}
$$

where

$$
L_{1} u=\varepsilon u^{\prime}(x)+a(x) u(x),
$$

$0<\varepsilon \ll 1$ is a perturbation parameter, $\lambda$ is a given parameter, $a(x) \geq \alpha>0$ and $f(x)$ $(x \in I), K_{1}(x, t)$ and $K_{2}(x, t)((x, t) \in I \times I)$ are sufficiently smooth functions.

In recent times, there has been an increased interest in numerical solutions of singularly perturbed integro-differential equations and various difference schemes have been presented for both singularly perturbed Volterra integro-differential equations (SPVIDEs) and singularly perturbed Fredholm integro-differential equations (SPFIDEs). In [30], by using Simpson quadrature rule and Richardson extrapolation, the order of convergence of the numerical scheme has been enhanced for SPVIDEs. Exponentially fitted difference scheme has been constructed on a Shishkin mesh for second order SPFIDEs in [11]. The stability and convergence of the difference scheme have been analyzed for SPVIDEs in [22]. Boundary value problems of SPFIDEs have been investigated in [9]. Authors in [40] have presented second-order discretization on a piecewise uniform mesh for SPVIDEs. The finite difference scheme with exponential coefficient has been established on a uniform mesh for SPFIDEs in [3]. In [33], for SPVIDEs, a fitted mesh finite difference technique with Richardson extrapolation has been applied on piecewise-uniform Shishkin mesh. SPVIDEs with delay arguments have been discretized in [5].

Our motivation in this article is to present reliable and robust numerical approach for solving SPVFIDEs on a Shishkin mesh.

This work is arranged as follows: Asymptotic estimations of the exact solution are introduced in Section 2. In Section 3, the finite difference scheme is constructed on a Shishkin mesh. Error approximations and convergence analysis are presented in Section 4. Experimental results with some examples are given in Section 5. The paper ends with "Discussion and conclusion" section.

\section{Properties of the exact solution}

Conveinent asymptotic estimations of the exact solution and its derivatives are given in this section.

Lemma 2.1 ([26]). Take into account the following initial-value problem

$$
\begin{aligned}
\varepsilon v^{\prime}(x)+a(x) v(x) & =F(x), 0<x<l, \\
v(0) & =A .
\end{aligned}
$$

Let $a(x) \geq \alpha>0, F(x) \in C(\bar{I}),|F(x)| \leq \mathcal{F}(x)$ and the function $\mathcal{F}(x)$ is nondecreasing. Then, the solution of the problem (2.1)-(2.2) satisfies that

$$
|v(x)| \leq|A|+\alpha^{-1} \mathcal{F}(x), 0<x<l .
$$


Lemma 2.2. We assume that

$$
a, f \in C^{1}[0, l], \quad \frac{\partial}{\partial x} K_{1}(x, t) \in[0, l]^{2}, \frac{\partial}{\partial x} K_{2}(x, t) \in[0, l]^{2}
$$

and

$$
\gamma=e^{\alpha^{-1} \bar{K}_{1} l} \alpha^{-1}|\lambda| \max _{0 \leq x \leq l} \int_{0}^{l}\left|K_{2}(x, t)\right| d t<1 .
$$

Then, the solution $u(x)$ of the problem (1.1)-(1.2) holds

$$
\|u\|_{\infty} \leq C_{0}
$$

and

where

$$
\left|u^{\prime}(x)\right| \leq C\left\{1+\frac{1}{\varepsilon} e^{\frac{-\alpha x}{\varepsilon}}\right\}, \quad x \in[0, l] .
$$

$$
C_{0}=(1-\gamma)^{-1}\left(|A|+\alpha^{-1}\|f\|_{\infty}\right) e^{\alpha^{-1} \bar{K}_{1} l}
$$

and

$$
\bar{K}_{1}=\max _{x \in[0, l]}\left|K_{1}(x, t)\right| .
$$

Proof. Firstly, we show the proof of (2.4). The equation (1.1) can be rewritten as follows

$$
\varepsilon u^{\prime}(x)+a(x) u(x)=F(x), 0<x<l
$$

where

$$
F(x)=f(x)-\int_{0}^{x} K_{1}(x, t) u(t) d t-\lambda \int_{0}^{l} K_{2}(x, t) u(t) d t .
$$

Then, we estimate (2.7) as the form

$$
|F(x)| \leq\|f\|_{\infty}+\bar{K}_{1} \int_{0}^{x}|u(t)| d t+|\lambda| \int_{0}^{l}\left|K_{2}(x, t)\right||u(t)| d t .
$$

Considering Lemma 2.1 for the equation (2.6), we obtain

$$
|u(x)| \leq \delta+\alpha^{-1} \bar{K}_{1} \int_{0}^{x}|u(t)| d t .
$$

where

$$
\delta=|A|+\alpha^{-1}\|f\|_{\infty}+\alpha^{-1}|\lambda| \int_{0}^{l}\left|K_{2}(x, t)\right||u(t)| d t .
$$

Applying the Gronwall's inequality to the inequality (2.8), we have

$$
|u(x)| \leq \delta \exp \left(\alpha^{-1} \bar{K}_{1} x\right) .
$$

We can write the inequality (2.9) clearly that

$$
\begin{gathered}
|u(x)| \leq\left(|A|+\alpha^{-1}\|f\|_{\infty}\right) \exp \left(\alpha^{-1} \bar{K}_{1} x\right) \\
+\alpha^{-1}|\lambda| \int_{0}^{l}\left|K_{2}(x, t)\right||u(t)| d t \exp \left(\alpha^{-1} \bar{K}_{1} x\right) .
\end{gathered}
$$

Modifying the relation (2.10), it is found that

$$
\|u\|_{\infty}\left(1-\alpha^{-1}|\lambda| \max _{0 \leq x \leq l} \int_{0}^{l}\left|K_{2}(x, t)\right| d t \exp \left(\alpha^{-1} \bar{K}_{1} l\right)\right)
$$




$$
\leq\left(|A|+\alpha^{-1}\|f\|_{\infty}\right) \exp \left(\alpha^{-1} \bar{K}_{1} l\right)
$$

which validates the estimation (2.4). Now, we prove (2.5). Then, we estimate $u^{\prime}(0)$. From (1.1), we have

$$
\left|u^{\prime}(0)\right| \leq \frac{1}{\varepsilon}\left(|f(0)|-|a(0)||A|-|\lambda| \int_{0}^{l}\left|K_{2}(0, t)\right||u(t)| d t\right) .
$$

Since $\left|K_{2}(x, t)\right| \leq \bar{K}_{2}$ and $|u| \leq C_{0}$, following inequality is written:

$$
\left|u^{\prime}(0)\right| \leq \frac{C}{\varepsilon} .
$$

By differentiating (2.6), we get

$$
\varepsilon v^{\prime}+a(x) v=F(x)
$$

with

$$
v(x)=u^{\prime}(x)
$$

Here

$$
F(x)=f^{\prime}(x)-a^{\prime}(x) u(x)-\int_{0}^{x} \frac{\partial}{\partial x} K_{1}(x, t) u(t) d t-K_{1}(x, x) u(x)-\lambda \int_{0}^{l} \frac{\partial}{\partial x} K_{2}(x, t) u(t) d t .
$$

Considering (2.3) and (2.4), we can write

$$
|F(x)| \leq C .
$$

From (2.12), we obtain

$$
u^{\prime}(x)=u^{\prime}(0) e^{-\frac{1}{\varepsilon} \int_{\xi}^{x} a(\tau) d \tau}+\frac{1}{\varepsilon} \int_{0}^{x} F(\xi) e^{-\frac{1}{\varepsilon} \int_{\xi}^{x} a(\tau) d \tau} d \xi .
$$

Consequently, owing to (2.11) and (2.13), the following expression is found:

$$
\left|u^{\prime}(x)\right| \leq \frac{C}{\varepsilon} e^{\frac{-\alpha x}{\varepsilon}}+\alpha^{-1}\|F\|_{\infty}\left(1-e^{\frac{-\alpha x}{\varepsilon}}\right)
$$

which implies (2.5). Therefore, the lemma is proven.

\section{Description of the difference scheme}

We denote by $\omega_{N}$ the non-uniform mesh on $[0, l]$

$$
\omega_{N}=\left\{0<x_{1}<x_{2}<\ldots<x_{N-1}<l, h_{i}=x_{i}-x_{i-1}\right\}
$$

and

$$
\bar{\omega}_{N}=\omega_{N} \cup\{x=0, x=l\} .
$$

We use some notation for the mesh functions. For any mesh function we defined on $\bar{\omega}_{N}$, we use

$$
v_{i}=v\left(x_{i}\right), v_{\bar{x}, i}=\frac{v_{i}-v_{i-1}}{h_{i}},\|v\|_{\infty}=\|v\|_{\infty, \bar{\omega}_{N}}=\max _{0 \leq i \leq N}\left|v_{i}\right| .
$$

We construct the difference scheme on Shishkin-type mesh for solving the problem (1.1)(1.2). For an even number $N$, we divide each of the subintervals $[0, \sigma]$ and $[\sigma, l]$ into $\frac{N}{2}$ equidistant subintervals. The transition point $\sigma$ is determined as

$$
\sigma=\min \left\{\frac{l}{2}, \alpha^{-1} \varepsilon \ln N\right\} .
$$


We use the notation $h^{(1)}$ for the mesh width in $[0, \sigma]$ and the notation $h^{(2)}$ for the width in $[\sigma, l]$. Hence, the mesh stepsizes hold

$$
\begin{gathered}
h^{(1)}=\frac{2 \sigma}{N}, \quad h^{(2)}=\frac{2(l-\sigma)}{N}, \\
h^{(1)} \leq l N^{-1}, \quad l N^{-1} \leq h^{(2)} \leq l N^{-1}, \quad h^{(1)}+h^{(2)}=2 l N^{-1} .
\end{gathered}
$$

$x_{i}$ node points are specified as

$$
\bar{\omega}_{N}=\left\{\begin{array}{c}
x_{i}=i h^{(1)}, \quad i=0,1, \ldots, \frac{N}{2}, x_{i} \in[0, \sigma] \\
x_{i}=\sigma+\left(i-\frac{N}{2}\right) h^{(2)}, \quad i=\frac{N}{2}+1, \ldots, N, x_{i} \in[\sigma, l] .
\end{array}\right.
$$

We start with the following integral identity for the equation (1.1):

$$
\begin{gathered}
\chi_{i}^{-1} h_{i}^{-1} \int_{x_{i-1}}^{x_{i}} \varepsilon u^{\prime}(x) \varphi_{i} d x+\chi_{i}^{-1} h_{i}^{-1} \int_{x_{i-1}}^{x_{i}} a(x) u(x) \varphi_{i} d x+\chi_{i}^{-1} h_{i}^{-1} \int_{x_{i-1}}^{x_{i}}\left[\int_{0}^{x} K_{1}(x, t) u(t) d t\right] \varphi_{i} d x \\
\quad+\chi_{i}^{-1} h_{i}^{-1} \lambda \int_{x_{i-1}}^{x_{i}}\left[\int_{0}^{l} K_{2}(x, t) u(t) d t\right] \varphi_{i} d x=\chi_{i}^{-1} h_{i}^{-1} \int_{x_{i-1}}^{x_{i}} f(x) \varphi_{i} d x,
\end{gathered}
$$

where the basis function

$$
\varphi_{i}(x)=e^{-\frac{a_{i}\left(x_{i}-x\right)}{\varepsilon}}, \quad i=1,2, \ldots, N .
$$

$\varphi_{i}(x)$ is the solution of the following problem:

$$
\begin{gathered}
-\varepsilon \varphi_{i}^{\prime}(x)+a_{i} \varphi_{i}(x)=0, \quad x_{i-1} \leq x \leq x_{i} \\
\varphi_{i}(x)=1 .
\end{gathered}
$$

For the first two term of (3.1), following relation is obtained:

$$
\begin{gathered}
h_{i}^{-1} \chi_{i}^{-1} \int_{x_{i-1}}^{x_{i}}\left[\varepsilon u^{\prime}(x)+a(x) u(x)\right] \varphi_{i}(x) d x=h_{i}^{-1} \chi_{i}^{-1} \int_{x_{i-1}}^{x_{i}}\left[\varepsilon u^{\prime}(x)+a\left(x_{i}\right) u(x)\right] \varphi_{i}(x) d x \\
+h_{i}^{-1} \chi_{i}^{-1} \int_{x_{i-1}}^{x_{i}}\left[a(x)-a\left(x_{i}\right)\right] u(x) \varphi_{i}(x) d x \\
=\varepsilon \vartheta_{i} u_{\bar{x}, i}+a_{i} u_{i}+R_{i}^{(1)}
\end{gathered}
$$

where

$$
\begin{gathered}
\vartheta_{i}=\frac{a_{i} \varrho_{i}}{1-e^{-a_{i} \varrho_{i}}} e^{-a_{i} \varrho_{i}}, \quad \varrho_{i}=\frac{h_{i}}{\varepsilon}, \\
R_{i}^{(1)}=h_{i}^{-1} \chi_{i}^{-1} \int_{x_{i-1}}^{x_{i}}\left[a(x)-a\left(x_{i}\right)\right] u(x) \varphi_{i}(x) d x
\end{gathered}
$$

and the $\chi_{i}$ coefficient

$$
\chi_{i}=h_{i}^{-1} \int_{x_{i-1}}^{x_{i}} \varphi_{i}(x) d x .
$$

For the right-side integral term of (3.1), we have

$$
h_{i}^{-1} \chi_{i}^{-1} \int_{x_{i-1}}^{x_{i}} f(x) \varphi_{i}(x) d x=f_{i}+R_{i}^{(2)}
$$


where

$$
R_{i}^{(2)}=h_{i}^{-1} \chi_{i}^{-1} \int_{x_{i-1}}^{x_{i}}\left[f(x)-f\left(x_{i}\right)\right] \varphi_{i}(x) d x .
$$

For the third term in left side of (3.1), using interpolating quadrature rules in [4], we find

$$
\chi_{i}^{-1} h_{i}^{-1} \int_{x_{i-1}}^{x_{i}} d x \varphi_{i}(x) \int_{0}^{x} K_{1}(x, t) u(t) d t=\int_{0}^{x} K_{1}\left(x_{i}, t\right) u(t) d t+R_{i}^{(3)}
$$

where

$$
R_{i}^{(3)}=-\chi_{i}^{-1} h_{i}^{-1} \int_{x_{i-1}}^{x_{i}} d x \varphi_{i}(x) \int_{x_{i-1}}^{x_{i}}\left(\int_{0}^{x} \frac{\partial}{\partial x} K_{1}(x, t) u(t) d t\right) d x .
$$

Using the right side rectangle rule to the right side of (3.5), we get

$$
\int_{0}^{x} K_{1}\left(x_{i}, t\right) u(t) d t+R_{i}^{(3)}=\sum_{j=1}^{i} h_{j} K_{1, i j} u_{j}+R_{i}^{(3)}+R_{i}^{(4)}
$$

where

$$
R_{i}^{(4)}=-\sum_{j=1}^{i} \int_{x_{j-1}}^{x_{j}}\left(\xi-x_{j-1}\right) \frac{\partial}{\partial \xi}\left(\int_{0}^{x} K_{1}(\xi, t) u(t) d t\right) d \xi .
$$

Eventually, for the fourth term in left side of (3.1), applying the interpolating quadrature rules in [4], it is found

$$
\chi_{i}^{-1} h_{i}^{-1} \lambda \int_{x_{i-1}}^{x_{i}} d x \varphi_{i}(x) \int_{0}^{l} K_{2}(x, t) u(t) d t=\lambda \int_{0}^{l} K_{2}\left(x_{i}, t\right) u(t) d t+R_{i}^{(5)}
$$

where

$$
R_{i}^{(5)}=-\chi_{i}^{-1} h_{i}^{-1} \lambda \int_{x_{i-1}}^{x_{i}} d x \varphi_{i}(x) \int_{x_{i-1}}^{x_{i}}\left(\int_{0}^{l} \frac{\partial}{\partial x} K_{2}(x, t) u(t) d t\right) d x .
$$

After, applying right side rectangle rule to the right side of (3.9), we have

$$
\lambda \int_{0}^{l} K_{2}\left(x_{i}, t\right) u(t) d t+R_{i}^{(5)}=\lambda \sum_{j=1}^{N} h_{j} K_{2, i j} u_{j}+R_{i}^{(5)}+R_{i}^{(6)}
$$

where

$$
R_{i}^{(6)}=-\lambda \sum_{j=1}^{N} \int_{x_{j-1}}^{x_{j}}\left(\xi-x_{j-1}\right) \frac{\partial}{\partial \xi}\left(\int_{0}^{x} K_{2}(\xi, t) u(t) d t\right) d \xi
$$

Combining (3.2), (3.4), (3.7) and (3.10), we can write the following difference scheme:

$$
\varepsilon \vartheta_{i} u_{\bar{x}, i}+a_{i} u_{i}+\sum_{j=1}^{i} h_{j} K_{1, i j} u_{j}+\lambda \sum_{j=1}^{N} h_{j} K_{2, i j} u_{j}+R_{i}=f_{i}, \quad i=1,2, \ldots, N,
$$

with remainder term

$$
R_{i}=\sum_{k=1}^{6} R_{i}^{(k)}
$$

By omitting the error term in (3.11), the following difference scheme is presented for the approximate solution:

$$
\varepsilon \vartheta_{i} y_{\bar{x}, i}+a_{i} y_{i}+\sum_{j=1}^{i} h_{j} K_{1, i j} y_{j}+\lambda \sum_{j=1}^{N} h_{j} K_{2, i j} y_{j}=f_{i}, \quad i=1,2, \ldots, N,
$$




$$
y_{0}=A,
$$

where $\vartheta_{i}$ is stated by (3.3).

\section{Error analysis}

In this section, the convergence of the proposed method is examined. The error function $z_{i}=y_{i}-u_{i}, i=0,1,2, \ldots, N$ is the solution of the following problem:

$$
\begin{gathered}
l z_{i}=R_{i}, i=0,1,2, \ldots, N, \\
z_{0}=0 .
\end{gathered}
$$

Lemma 4.1 ([26]). Consider the following difference problem

$$
\begin{gathered}
\varepsilon \vartheta_{i} v_{\bar{x}, i}+a_{i} v_{i}=F_{i}, i=0,1,2, \ldots, N, \\
v_{0}=A .
\end{gathered}
$$

Let $\left|F_{i}\right| \leq \mathcal{F}_{i}$ and the function $\mathcal{F}_{i}$ be nondecreasing. Then, the solution of (4.1)-(4.2) holds

$$
\left|v_{i}\right| \leq|A|+\alpha^{-1} \mathcal{F}_{i}, i=0,1,2, \ldots, N .
$$

Lemma 4.2. Let $y_{i}$ be the solution of (3.13)-(3.14). If

$$
\bar{\gamma}=\alpha^{-1}|\lambda| e^{\alpha^{-1} \bar{K}_{1} x_{i}} \max _{1 \leq i \leq N} \sum_{j=1}^{N} h_{j}\left|K_{2, i j}\right|<1
$$

then, for the solution of the difference problem (3.13)-(3.14), the following estimate is satisfied:

$$
\|y\|_{\infty} \leq(1-\bar{\gamma})^{-1}\left(|A|+\alpha^{-1}\|f\|_{\infty}\right) e^{\alpha^{-1} \bar{K}_{1} x_{i}} .
$$

Proof. The difference scheme (3.13) can be rewritten in the form

$$
\varepsilon \vartheta_{i} y_{\bar{t}, i}+a_{i} y_{i}=F_{i}, i=1,2, \ldots, N-1
$$

where

$$
F_{i}=f_{i}-\sum_{j=1}^{i} h_{j} K_{1, i j} y_{j}-\lambda \sum_{j=1}^{N} h_{j} K_{2, i j} y_{j}
$$

From (4.3), we get

$$
\left|F_{i}\right| \leq\|f\|_{\infty}+\sum_{j=1}^{i} h_{j}\left|K_{1, i j}\right|\left|y_{j}\right|+|\lambda| \sum_{j=1}^{N} h_{j}\left|K_{2, i j}\right|\left|y_{j}\right| .
$$

Moreover, applying Lemma 4.1. to (4.4), we have

$$
\begin{aligned}
\left|y_{i}\right| \leq|A|+\alpha^{-1}\|f\|_{\infty} & +\alpha^{-1} \bar{K}_{1} \sum_{j=1}^{i} h_{j}\left|y_{j}\right|+\alpha^{-1}|\lambda| \sum_{j=1}^{N} h_{j}\left|K_{2, i j}\right|\left|y_{j}\right| \\
& \leq \bar{\delta}+\alpha^{-1} \bar{K}_{1} \sum_{j=1}^{i} h_{j}\left|y_{j}\right|
\end{aligned}
$$

where

$$
\bar{\delta}=|A|+\alpha^{-1}\|f\|_{\infty}+\alpha^{-1}|\lambda| \sum_{j=1}^{N} h_{j}\left|K_{2, i j}\right|\left|y_{j}\right| .
$$

Applying the difference analogue of Gronwall's inequality to the relation (4.5), we obtain

$$
\left|y_{i}\right| \leq \bar{\delta} e^{\alpha^{-1} \bar{K}_{1} x_{i}}
$$


Then, it can be written obviously that

$$
\|y\|_{\infty} \leq\left(|A|+\alpha^{-1}\|f\|_{\infty}\right) e^{\alpha^{-1} \bar{K}_{1} x_{i}}+\alpha^{-1}|\lambda| e^{\alpha^{-1} \bar{K}_{1} x_{i}} \max _{1 \leq i \leq N} \sum_{j=1}^{N} h_{j}\left|K_{2, i j}\right|\|y\|_{\infty} .
$$

To estimate the error function, by rewriting $A=0, f=R$ and $y=z$ in (4.6), it is found that

$$
\|z\|_{\infty} \leq(1-\bar{\gamma})^{-1} \alpha^{-1}\|R\|_{\infty} e^{\alpha^{-1} \bar{K}_{1} x_{i}}
$$

Thus, the proof of the lemma is fulfilled.

Lemma 4.3. Under the conditions of Lemma 2.2 and $\frac{\partial K_{1}(x, t)}{\partial t} \in[0, l]^{2}, \frac{\partial K_{2}(x, t)}{\partial t} \in[0, l]^{2}$, the error term $R_{i}$ holds

$$
\|R\|_{\infty} \leq C N^{-1} \ln N
$$

Proof. Using the mean value theorem, we have

$$
\begin{gathered}
\left|a(x)-a\left(x_{i}\right)\right|=\left|a^{\prime}\left(\eta_{i}\right)\right|\left|x-x_{i}\right|, \quad \eta_{i} \in\left(x_{i}, x\right) \\
\leq C h_{i}
\end{gathered}
$$

Therefore, we find

$$
\left|R_{i}^{(1)}\right| \leq \chi_{i}^{-1} h_{i}^{-1} \int_{x_{i-1}}^{x_{i}} C h_{i} \varphi_{i}(x) d x \leq C h_{i} \chi_{i}^{-1} h_{i}^{-1} \int_{x_{i-1}}^{x_{i}} \varphi_{i}(x) d x \leq C h_{i} .
$$

By the same way, it can be shown that $\left|R_{i}^{(2)}\right| \leq C h_{i}$. For the remainder term $R_{i}^{(3)}$, applying Leibnitz rule to the integral term in (3.6), we have

$$
R_{i}^{(3)}=-\chi_{i}^{-1} h_{i}^{-1} \int_{x_{i-1}}^{x_{i}} d x \varphi_{i}(x) \int_{x_{i-1}}^{x_{i}}\left(K_{1}(x, x) u(x)+\frac{d}{d x} \int_{0}^{x} K_{1}(x, t) u(t) d t\right) d x
$$

Thus, the following relation can be written:

$$
\begin{gathered}
\left|R_{i}^{(3)}\right| \leq \int_{x_{i-1}}^{x_{i}}\left(\left|K_{1}(x, x)\right||u(x)|+\left|\int_{0}^{x} \frac{\partial}{\partial x} K_{1}(x, t) u(t) d t\right|\right) d x \\
\left|R_{i}^{(3)}\right| \leq C h_{i} .
\end{gathered}
$$

In a similar way, $\left|R_{i}^{(5)}\right| \leq C h_{i}$ is found. For the error term $R_{i}^{(4)}$, using Leibnitz rule for integral term in (3.8), we obtain

$$
\begin{aligned}
\left|R_{i}^{(4)}\right| \leq \sum_{j=1}^{i} \int_{x_{i-1}}^{x_{i}}\left(\xi-x_{j-1}\right)\left[\left|K_{1}(\xi, x) u(x)\right|+\int_{0}^{x}\left|\frac{\partial}{\partial \xi} K_{1}(\xi, t) u(t) d t\right|\right] d \xi \\
\leq \int_{0}^{l}\left(\xi-x_{j-1}\right)\left[\left|K_{1}(\xi, x) u(x)\right|+\int_{0}^{x}\left|\frac{\partial}{\partial \xi} K_{1}(\xi, t) u(t) d t\right|\right] d \xi \\
\leq C\left\{h_{i}+\int_{x_{i-1}}^{x_{i}}\left|u^{\prime}(x)\right| d x\right\} .
\end{aligned}
$$

Similarly,

$$
\left|R_{i}^{(6)}\right| \leq C\left\{h_{i}+\int_{x_{i-1}}^{x_{i}}\left|u^{\prime}(x)\right| d x\right\}
$$


According to the node points of Shishkin mesh, we take the following estimations. Initially, considering the first case $\sigma=\frac{l}{2}$ and $\frac{l}{2}<\alpha^{-1} \varepsilon \ln N$, we find $h^{(1)}=h^{(2)}=h=l N^{-1}$. Hence, we evaluate $R_{i}^{(k)}$ for $k=1,2,3,5$. Now, we estimate the remainder term $R_{i}^{(4)}$.

$$
\begin{aligned}
\left|R_{i}^{(4)}\right| & \leq C\left\{h+\int_{x_{i-1}}^{x_{i}}\left|u^{\prime}(x)\right| d x\right\} \leq C\left\{h+\int_{x_{i-1}}^{x_{i}} \frac{1}{\varepsilon} e^{-\frac{\alpha x}{\varepsilon}} d x\right\} \\
& \leq C\left\{h+\varepsilon^{-1} \int_{x_{i-1}}^{x_{i}} e^{-\frac{\alpha x}{\varepsilon}} d x\right\}=2 \alpha^{-1} N^{-1} \ln N .
\end{aligned}
$$

Likewise, we obtain $R_{i}^{(6)}=2 \alpha^{-1} N^{-1} \ln N$. In the second case, for the interval $[\sigma, l]$, we have the inequality

$$
\begin{gathered}
\left|R_{i}^{(4)}\right| \leq C\left\{h+\int_{x_{i-1}}^{x_{i}}\left|u^{\prime}(x)\right| d x\right\} \leq C\left\{h+\varepsilon^{-1} \int_{x_{i-1}}^{x_{i}} e^{-\frac{\alpha x}{\varepsilon}} d x\right\} \\
\leq C\left\{h+\alpha^{-1}\left(e^{-\frac{\alpha x_{i-1}}{\varepsilon}}-e^{-\frac{\alpha x_{i}}{\varepsilon}}\right)\right\} .
\end{gathered}
$$

Since $x_{i}=\alpha^{-1} \varepsilon \ln N+\left(i-\frac{N}{2}\right) h$, we can write

$$
\begin{gathered}
e^{-\frac{\alpha x_{i-1}}{\varepsilon}}-e^{-\frac{\alpha x_{i}}{\varepsilon}}=\frac{1}{N} e^{\frac{-\alpha\left(i-1-\frac{N}{2}\right) H}{\varepsilon}}\left(1-e^{-\frac{\alpha H}{\varepsilon}}\right)<N^{-1} \\
\left|R_{i}^{(4)}\right| \leq C N^{-1} .
\end{gathered}
$$

For the interval $[0, \sigma]$, if we take $\sigma=\alpha^{-1} \varepsilon \ln N$, we get

$$
\begin{gathered}
\left|R_{i}^{(4)}\right| \leq C\left(1+\varepsilon^{-1}\right) \frac{\alpha^{-1} \varepsilon \ln N}{N / 2}, \\
\left|R_{i}^{(4)}\right| \leq C N^{-1} \ln N
\end{gathered}
$$

Similarly, we find

$$
\left|R_{i}^{(6)}\right| \leq C N^{-1} \ln N
$$

Thus, substituting the estimations of all remainder terms in (3.12), we obtain

$$
\left|R_{i}\right| \leq C N^{-1} \ln N
$$

Theorem 4.4. Let $u$ be the solution of (1.1)-(1.2) and $y$ be the solution of (3.13)-(3.14). Then, the following estimate is satisfied:

$$
\|y-u\|_{\infty, \bar{\omega}_{N}} \leq C N^{-1} \ln N .
$$

Proof. This follows immediately by combining of previous two lemmas. 


\section{Illustrative examples}

In this section, theoretical results are tested on two samples. In this context, we use the following iteration for solving discretization (3.13)-(3.14):

$$
\begin{aligned}
& y_{i}^{(n)}=\frac{\varepsilon \vartheta_{i} y_{i-1}^{(n)}+h_{i} f_{i}-h_{i}\left(\sum_{j=1}^{i} h_{j} K_{1, i j} y_{j}^{(n-1)}+\lambda \sum_{j=1}^{N} h_{j} K_{2, i j} y_{j}^{(n-1)}\right)}{\varepsilon \vartheta_{i}+h_{i} a_{i}}, \\
& y_{0}^{(n)}=A \text {. }
\end{aligned}
$$

Example 1: We take into account the following singularly perturbed Volterra-Fredholm equation:

$$
\varepsilon u^{\prime}+u+\int_{0}^{x} x u(t) d t+\int_{0}^{1} u(t) d t=e^{\frac{-x}{\varepsilon}}\left(-\varepsilon^{2}+\varepsilon+1\right)+\varepsilon x-\varepsilon e^{\frac{-1}{\varepsilon}}+\varepsilon
$$

subject to initial condition

$$
u(0)=1 .
$$

The exact solution of this problem is $u(x)=e^{\frac{-x}{\varepsilon}}$. Error approximations are computed as

$$
e^{N}=\left|y_{i}-u_{i}\right|
$$

where $u_{i}$ is the exact solution and $y_{i}$ is approximate solution. Besides, the order of convergence is defined as follows

$$
p^{N}=\frac{\ln \left(e^{N} / e^{2 N}\right)}{\ln 2} .
$$

Experimental datas are displayed in Table 1.

Table 1. Maximum pointwise errors $e^{N}$ and order of convergence $p^{N}$ on $\bar{\omega}_{N}$

\begin{tabular}{cccccc}
\hline$\varepsilon$ & $N=64$ & $N=128$ & $N=256$ & $N=512$ & $N=1024$ \\
\hline $2^{-2}$ & 0.00817305 & 0.00399709 & 0.00197592 & 0.00098227 & 0.00048971 \\
& 1.031 & 1.016 & 1.008 & 1.004 & 1.002 \\
$2^{-4}$ & 0.00918949 & 0.00426125 & 0.00204326 & 0.00099927 & 0.00049398 \\
& 1.108 & 1.060 & 1.032 & 1.016 & 1.023 \\
$2^{-6}$ & 0.01477887 & 0.00738994 & 0.00369472 & 0.00184726 & 0.00092348 \\
& 0.999 & 1.001 & 1.001 & 1.002 & 1.005 \\
$2^{-8}$ & 0.01531296 & 0.00767148 & 0.00383674 & 0.00191787 & 0.00095693 \\
& 0.997 & 0.999 & 1.004 & 1.003 & 1.008 \\
$2^{-10}$ & 0.01554171 & 0.00770116 & 0.00384749 & 0.00192375 & 0.00096087 \\
& 1.013 & 1.001 & 1.000 & 1.003 & 1.001 \\
& & & & & \\
$e^{N}$ & 0.01554171 & 0.00770116 & 0.00384749 & 0.00192375 & 0.00096087 \\
$p^{N}$ & 0.997 & 0.999 & 1.000 & 1.002 & 1.001 \\
\hline
\end{tabular}

Example 2: Consider the another problem

$$
\begin{gathered}
\varepsilon u^{\prime}+\left(3 x^{2}+1\right) u+\int_{0}^{x} t u(t) d t+\frac{1}{2} \int_{0}^{1}(1-x t) u(t) d t=e^{\frac{-x}{\varepsilon}}(x+\varepsilon), \\
u(0)=1 .
\end{gathered}
$$

The exact solution of this problem is unknown. Since the exact solution is unknown, we apply the double-mesh technique. The maximum pointwise errors are remarked by

$$
e^{N}=\left|y_{i}^{N}-y_{i}^{2 N}\right|
$$


and the convergence rates are specified as follows

$$
p^{N}=\frac{\ln \left(e^{N} / e^{2 N}\right)}{\ln 2} .
$$

The computational results are tabulated in Table 2 .

\begin{tabular}{cccccc}
\multicolumn{6}{c}{ Table 2. Maximum pointwise errors $e^{N}$ and order of convergence $p^{N}$ on $\bar{\omega}_{N}$} \\
\hline$\varepsilon$ & $N=64$ & $N=128$ & $N=256$ & $N=512$ & $N=1024$ \\
\hline $2^{-2}$ & 0.00811413 & 0.00398210 & 0.00197214 & 0.00098132 & 0.00048947 \\
& 1.026 & 1.014 & 1.007 & 1.003 & 1.019 \\
$2^{-4}$ & 0.00897773 & 0.00420438 & 0.00202853 & 0.00099552 & 0.00049304 \\
& 1.094 & 1.051 & 1.026 & 1.013 & 1.030 \\
$2^{-6}$ & 0.01054824 & 0.00529412 & 0.00263706 & 0.00131833 & 0.00065826 \\
& 0.994 & 1.005 & 1.000 & 1.002 & 1.006 \\
$2^{-8}$ & 0.01383275 & 0.00696637 & 0.00348319 & 0.00174159 & 0.00087080 \\
& 0.989 & 1.000 & 1.000 & 1.001 & 1.002 \\
$2^{-10}$ & 0.01509617 & 0.00754809 & 0.00376404 & 0.00187702 & 0.00093510 \\
& 1.000 & 1.003 & 1.003 & 1.005 & 1.011 \\
& & & & & \\
$e^{N}$ & 0.01509617 & 0.00754809 & 0.00376404 & 0.00187702 & 0.00093510 \\
$p^{N}$ & 0.989 & 1.000 & 1.000 & 1.001 & 1.002 \\
\hline
\end{tabular}

From Tables 1-2, it can be observed that almost first-order convergence is acquired for different values of the perturbation parameter and mesh stepsize. This shows that the numerical applications accordance with the theory.

\section{Discussion and conclusion}

A new difference scheme was introduced by using exponential basis functions and interpolating quadrature rules to get the numerical solution of SPVIDEs. The difference scheme was constructed on a Shishkin mesh. Error analysis of the method was completed and two test problems were solved. The obtained outcomes were shown in Tables 1-2 and the order of uniform convergence was found as $O\left(N^{-1} \ln N\right)$. The computed results show that the proposed method is stable and very effective for solving these problems. It can also be applied to partial and fractional types of integro-differential equations for future investigations.

\section{References}

[1] A. Abubakar and O.A. Taiwo, Integral collocation approximation methods for the numerical solution of high-orders linear Fredholm-Volterra integro-differential equations, American Journal of Computational and Applied Mathematics, 4(4), 111-117, 2014.

[2] N.I. Acar and A. Daşcioğlu, A projection method for linear Fredholm-Volterra integro differential equations, J. Taibah Univ. Sci. 13 (1), 644-650, 2019.

[3] G.M. Amiraliyev, M.E. Durmaz and M. Kudu, Uniform convergence results for singularly perturbed Fredholm integro-differential equation, J. Math. Anal. 9 (6), 55-64, 2018.

[4] G.M. Amiraliyev and Y.D. Mamedov, Difference schemes on the uniform mesh for singularly perturbed pseudo-parabolic equations, Turk. J. Math. 19, 207-222, 1995.

[5] G.M. Amiraliyev, Ö. Yapman and M. Kudu, A fitted approximate method for a Volterra delay-integro-differential equation with initial layer, Hacet. J. Math. Stat. 48 (5), 1417-1429, 2019. 
[6] M.M. Arjunan and S. Selvi, Existence results for impulsive mixed Volterra-Fredholm integro-differential inclusions with nonlocal conditions, Int. J. Math. Sci. Appl. 1 (2), 101-119, 2015.

[7] J. Chen, M. He and Y. Huang, A fast multiscale Galerkin method for solving secondorder linear Fredholm integro-differential equation with Dirichlet boundary conditions, J. Comput. Appl. Math. 364 (1), Article ID 112352, 2020.

[8] M. Çakır, B. Güneş and H. Duru, A novel computational method for solving nonlinear Volterra integro-differential equation, Kuwait J. Sci. 48 (1), 31-40, 2021.

[9] E. Çimen and M. Çakır, A uniform numerical method for solving singularly perturbed Fredholm integro-differential problem, Comput. Appl. Math. 40, Article number 42, 2021.

[10] E.H. Doha, M.A. Abdelkawy, A.Z.M. Amin and D. Baleanu, Shifted Jacobi spectral collocation method with convergence analysis for solving integro-differential equations and system of integro-differential equations, Nonlinear Anal. Model. Control 24 (3), 332-352, 2019.

[11] M.E. Durmaz and G.M. Amiraliyev, A robust numerical method for a singularly perturbed Fredholm integro-differential equation, Mediterr. J. Math. 18, Article number 24, 2021.

[12] S.M. El- Sayed, D. Kaya and S. Zarea, The decomposition method applied to solve high-order linear Volterra-Fredholm integro-differential equations, Int. J. Nonlinear Sci. Numer. Simul. 5 (2), 105-112, 2004.

[13] A.I. Fairbairn and M.A. Kelmanson, Error analysis of a spectrally accurate Volterratransformation method for solving 1-D Fredholm integro-differential equations, Int. J. Mech. Sci. textbf144, 382-391, 2018.

[14] M. Fathy, M. El-Gamel and M.S. El-Azab, Legendre-Galerkin method for the linear Fredholm integro-differential equations, Appl. Math. Comput. 243, 789-800, 2014.

[15] M. Ghasemi, M. Fardi and R.K. Ghaziani, Solution of system of the mixed VolterraFredholm integral equations by an analytical method, Math. Comput. Model. 58, 15221530, 2013.

[16] M. Gülsu, Y. Öztürk and M. Sezer, A new collocation method for solution of mixed linear integro-differential-difference equations, Appl. Math. Comput. 216, 2183-2198, 2010 .

[17] A.A. Hamoud and K.P. Ghadle, The reliable modified of Adomian Decomposition method for solving integro-differential equations, J. Chungcheong Math. Soc. 32 (4), 409-420, 2019.

[18] A.A. Hamoud, K.H. Hussain, N.M. Mohammed and K.P. Ghadle, Solving Fredholm integro-differential equations by using numerical techniques, Nonlinear Funct. Anal. Appl. 24 (3), 533-542, 2019.

[19] A.A. Hamoud, N.M. Mohammed and K.P. Ghadle, Solving mixed Volterra-Fredholm integro differential equations by using HAM, Turk. J. Math. Comput. Sci. 12 (1), $18-25,2020$.

[20] E. Hesameddini and M. Shahbazi, Solving multipoint problems with linear VolterraFredholm integro-differential equations of the neutral type using Bernstein polynomials method, Appl. Numer. Math. 136, 122-138, 2019.

[21] M. Heydari, Z. Avazzadeh and G.B. Loghmani, Chebyshev cardinal functions for solving Volterra-Fredholm integro-differential equations using operational matrices, Iran. J. Sci. Technol. Trans. A Sci. 36 (1), 13-24, 2012.

[22] B.C. Iragi and J.B. Munyazaki, A uniformly convergent numerical method for a singularly perturbed Volterra integro-differential equation, Int. J. Comput. Math. 97 (4), 759-771, 2020. 
[23] K. Issa and F. Salehi, Approximate solution of perturbed Volterra-Fredholm integrodifferential equations by Chebyshev-Galerkin method, J. Math. 2017 Article ID 8213932, 2017.

[24] A.A. Jalal, N.A. Sleman and A.I. Amen, Numerical methods for solving the system of Volterra-Fredholm integro-differential equations, ZANCO J. Pure Appl. Sci. 31 (2), 25-30, 2019.

[25] K.D. Kucche and M.B. Dhakne, On existence results and qualitative properties of mild solution of semilinear mixed Volterra-Fredholm functional integro-differential equations in Banach spaces, Appl. Math. Comput. 219, 10806-10816, 2013.

[26] M. Kudu, I. Amirali and G.M. Amiraliyev, A finite difference method for a singularly perturbed delay integro-differential equations, J. Comput. Appl. Math. textbf308, 379390, 2016.

[27] K. Kumar and R. Kumar, Existence of solutions of quasilinear mixed VolterraFredholm integro differential equations with nonlocal conditions, Journal Differential Equations and Control Processes, 3, 77-84, 2013.

[28] A.H. Mahmood and L.H. Sadoon, Existence of a solution of certain Volterra-Fredholm integro-differential equations, J. Educ. Sci.25(3), 62-67, 2012.

[29] D.A. Maturi and E.A. Simbawa, The modified Decomposition method for solving Volterra-Fredholm integro-differential equations using Maple, Int. J. GEOMATE 18 (67), 84-89, 2020.

[30] N.A. Mbroh, S.C.O. Noutchie and R.Y.M. Massoukou, A second order finite difference scheme for singularly perturbed Volterra integro-differential equation. Alex. Eng. J. 59, 2441-2447, 2020.

[31] A.K.O. Mezaal, Efficient approximate method for solutions of linear mixed VolterraFredholm integro differential equations, Al-Mustansiriyah J. Sci. 27 (1), 58-61, 2016.

[32] E.H. Ouda, S. Shibab and M. Rasheed, Boubaker wavelets functions for solving higher order integro-differential equations, J. Southwest Jiaotong Univ. 55 (2), 2020.

[33] A. Panda, J. Mohapatra and I. Amirali, A second-order post-processing technique for singularly perturbed Volterra integro-differential equations, Mediterr. J. Math. 18, Article Number 231, 2021.

[34] B. Raftari, Numerical solutions of the linear Volterra integro-differential equations: Homotopy perturbation method and finite difference method, World Appl. Sci. J. 9, 7-12, 2010.

[35] M.A. Ramadan and M.R. Ali, Numerical solution of Volterra-Fredholm integral equations using Hybrid orthonormal Bernstein and Block-Pulse functions, Asian Res. J. Math. 4 (4), 1-14, 2017.

[36] S. Shahmorad, Numerical solution of the general form linear Fredholm-Volterra integro-differential equations by the Tau method with an error estimation, Appl. Math. Comput. 167, 1418-1429, 2005.

[37] A. Tari, M.Y. Rahimi, S. Shahmorad and F. Talati, Development of the Tau method for the numerical solution of two-dimensional linear Volterra integro-differential equations, Comput. Methods Appl. Math. 9 (4), 421-435, 2009.

[38] K. Wang and Q. Wang, Lagrange collocation method for solving Volterra-Fredholm integral equations, Appl. Math. Comput. 219 (21), 10434-10440, 2013.

[39] S. Yalçınbaş, M. Sezer and H.H. Sorkun, Legendre polynomial solutions of high-order linear Fredholm integro-differential equations, Appl. Math. Comput. 210, 334-349, 2009.

[40] Ö. Yapman and G.M. Amiraliyev, A novel second order fitted computational method for a singularly perturbed Volterra integro-differential equation, Int. J. Comput. Math. 97 (6), 1293-1302, 2020.

[41] A. Zanib and J. Ahmad, Variational iteration method for mixed type integrodifferential equations, Math. Theory Model. (IISTE), 6 (8), 1-7, 2016. 